Supporting Information

\title{
A Versatile Imaging and Therapeutic Platform Based on Dual-Band Luminescent Lanthanide Nanoparticles toward Tumor Metastasis Inhibition
}

Yang Li, ${ }^{\dagger, \S}$ Jinglong Tang,,${ }^{\dagger} \S$ Dong-Xu Pan,${ }^{\dagger}$ Ling-Dong Sun, ${ }^{\dagger} * *$ Chunying Chen, ${ }^{\ddagger} * *$ Ying Liu, ${ }^{\star}$ Ye-Fu Wang, ${ }^{\dagger}$ Shuo Shi ${ }^{\dagger}$ and Chun-Hua Yan ${ }^{\dagger} * *$

${ }^{\dagger}$ Beijing National Laboratory for Molecular Sciences, State Key Laboratory of Rare Earth Materials Chemistry and Applications, PKU-HKU Joint Laboratory in Rare Earth Materials and Bioinorganic Chemistry, College of Chemistry and Molecular Engineering, Peking University, Beijing 100871, China

E-mail: sun@pku.edu.cn; yan@pku.edu.cn

$¥$ CAS Key Laboratory for Biomedical Effects of Nanomaterials and Nanosafety \& CAS Center for Excellence in Nanoscience, National Center for Nanoscience and Technology of China, Beijing 100190, China

E-mail: chenchy@nanoctr.cn

$\S$ These authors contributed equally to this work. 
Materials. Oleic acid (OA; >90\%, Sigma-Aldrich), oleylamine (OM; >80\%, Acros), 1-octadecene (ODE; >90\%, Acros), trifluoroacetic acid (99\%, Acros), trifluoroacetic acid sodium salt (99\%, Acros), ethanol (AR), cyclohexane (AR), dimethylformamide (DMF, AR), nitrosonium tetrafluoroborate $\left(\mathrm{NOBF}_{4} ; 98 \%\right.$, Alfa Aesar), polyethylenimine (PEI; branched, $\mathrm{Mn} \sim 10000$ by GPC, Aldrich), rose bengal (RB; 90\%, Aldrich), bromohexanoic acid (HA-Br; 98\%, J \& K Chemical), 1,3diphenylisobenzofuran (DPBF, 97\%, Aldrich), 1-ethyl-3-(3-dimethylaminopropyl) carbodiimide (EDC; 99\%, Shanghai Mepop Co., Ltd.), and N-hydroxysulfosuccinimide sodium salt (NHS; TCI). All the reagents were used as received without further purification. The water used in this study was deionized by a milli-Q Plus system. All the organic solvents were analytical grade and used without further purification.

Instruments. Samples dispersed in cyclohexane for transmission electron microscopy (TEM) analysis were prepared by drying a drop of nanoparticle dispersion in cyclohexane on amorphous carbon-coated copper grids. Samples dispersed in water for TEM analysis were prepared by dropping $7 \mu \mathrm{L}$ of the samples on the plasma-treated amorphous carbon-coated copper grids for $30 \mathrm{~s}$, and then a piece of filter paper strip was used to draw the residual liquid away. TEM characterization was performed with a JEOL JEM-2100 TEM operated at $200 \mathrm{kV}$. UC emission and near-infrared (NIR) emission spectra were measured using an infrared fluorescence spectrometer (Nanolog FL3-2iHR, HORIBA JOBIN YVON), of UC emission measurement with a G2B3 shortpass filter, and NIR emission measurement with an $830 \mathrm{~nm}$ long pass filter. Lasers for $808 \mathrm{~nm}$ and $980 \mathrm{~nm}$ excitation are high power multimode pump lasers (Hi-tech Optoelectronic Co. Ltd., with a maximum power $5.0 \mathrm{~W}$ for both lasers). Laser power was measured with an optical power meter. Absorption spectra were measured by UVVisible spectrophotometer (190 - 1100 nm, Lambda35, Perkin Elmer). Time-resolved emission spectra were tested with a customized UV to mid-infrared steady-state and phosphorescence lifetime spectrometer (FSP920-C, Edinburgh) equipped with a tunable midband OPO pulse laser as excitation source $(410-2400 \mathrm{~nm}, 10 \mathrm{~Hz}$, pulse width $\leq 5 \mathrm{~ns}$, Vibrant 355II, OPOTEK). FT-IR spectra were tested by Fourier transform infrared spectrometer $\left(4000-400 \mathrm{~cm}^{-1}\right.$, VECTOR22, Bruker). The hydrodynamic diameter and zeta potential analysis were carried out on a nanoparticle analyzer (SZ100, HORIBA JOBIN YVON). ICP-AES results were obtained by Inductively Coupled Plasma-Atomic Emission Spectrometer (PROFILE SPEC, Leeman). The absorbance of cytotoxicity was measured by the microplate reader (Tecan, Durham, USA). The fluorescent images were obtained by the fluorescent microscopy (Olympus IX71). The histopathological images were obtained by the EVOS microscopy (Life technology Inc.). The MRI was obtained by the MR scanner (BioSpec70/20USR, Bruker, MA, USA). 


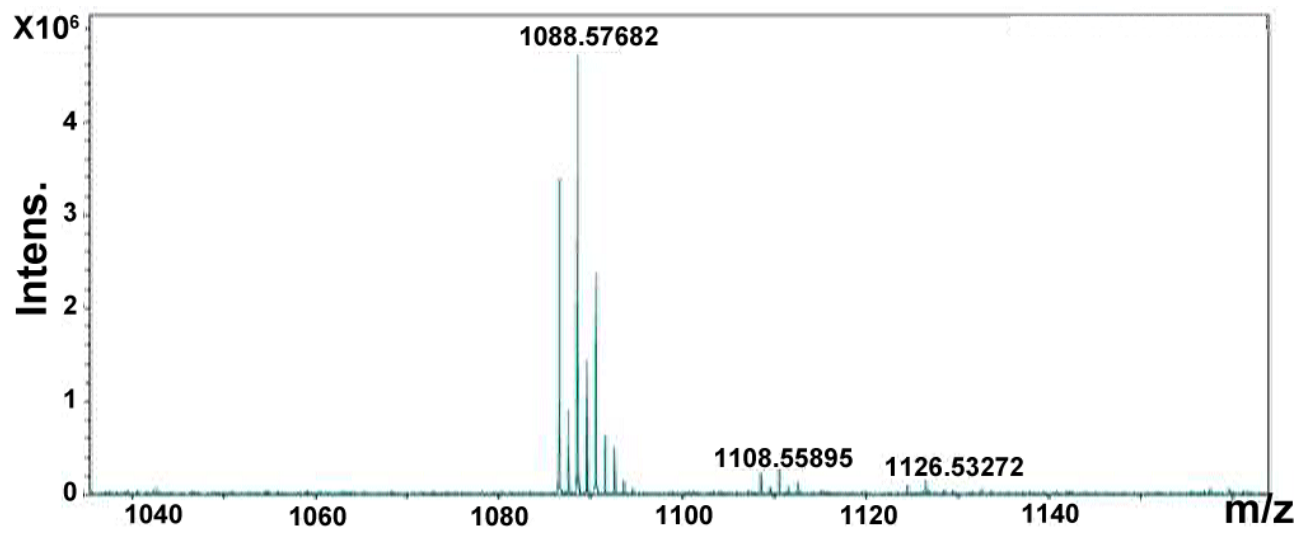

Figure S1. Mass spectrum of $\mathrm{RB}\left(\mathrm{CH}_{2}\right)_{5} \mathrm{COOH}$.

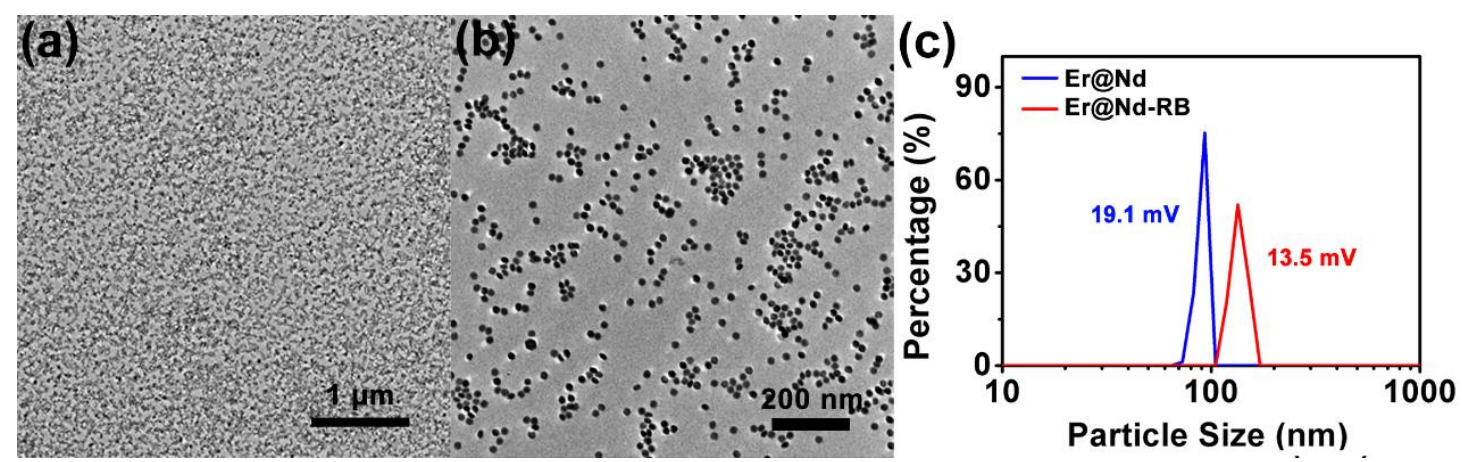

Figure S2. (a) TEM image, and (b) high-magnified TEM image of the Er@Nd-RB conjugates. (c) Hydrodynamic diameter and zeta potential of the Er@Nd NPs and $\mathrm{Er} @ \mathrm{Nd}-\mathrm{RB}$ conjugates, respectively. 


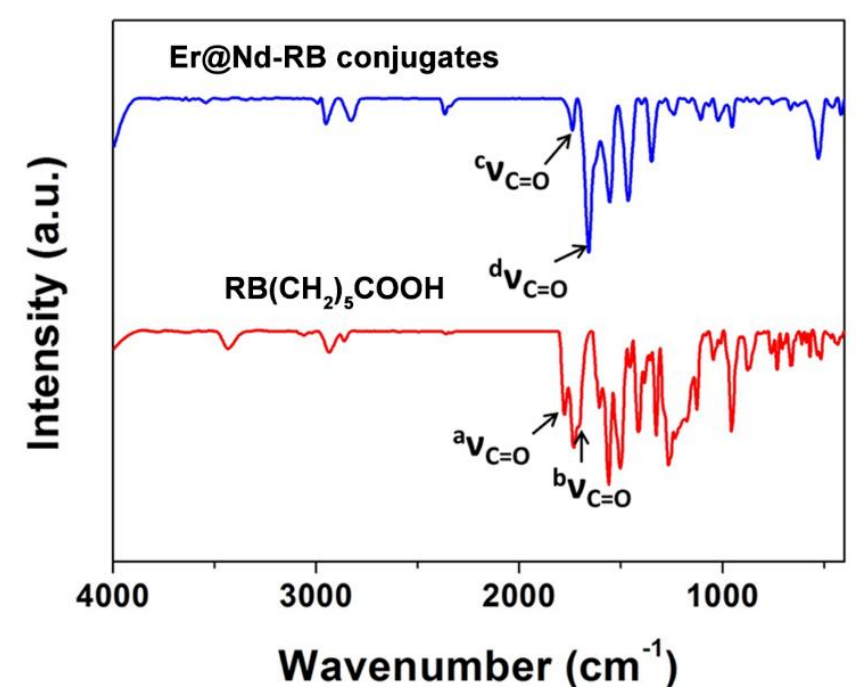

Figure S3. FT-IR spectra of the $\mathrm{RB}\left(\mathrm{CH}_{2}\right)_{5} \mathrm{COOH}$ and $\mathrm{Er} @ \mathrm{Nd}-\mathrm{RB}$ conjugates. ${ }^{\mathrm{a}-\mathrm{d}} \mathrm{C}=\mathrm{O}$ represent for carboxylic acid, ketone, ester, amide carbonyl groups, respectively.

For $\mathrm{RB}\left(\mathrm{CH}_{2}\right)_{5} \mathrm{COOH}$ molecules, $\mathrm{C}=\mathrm{O}$ stretching vibration mode of the carboxylic acid group locates at $1776 \mathrm{~cm}^{-1} .1705 \mathrm{~cm}^{-1}$ and $1743 \mathrm{~cm}^{-1}$ absorptions represent for $\mathrm{C}=\mathrm{O}$ stretching vibration modes of the ketone group and the ester group, respectively. After conjugating $\mathrm{RB}\left(\mathrm{CH}_{2}\right)_{5} \mathrm{COOH}$ with $\mathrm{PEI}$, stretching vibration signal of carboxylic acid carbonyl disappeared, and a new absorption appeared at $1656 \mathrm{~cm}^{-1}$, which represents for the $\mathrm{C}=\mathrm{O}$ stretching vibration mode of the amine group, confirming the successful reaction of $\mathrm{RB}\left(\mathrm{CH}_{2}\right)_{5} \mathrm{COOH}$ to PEI.

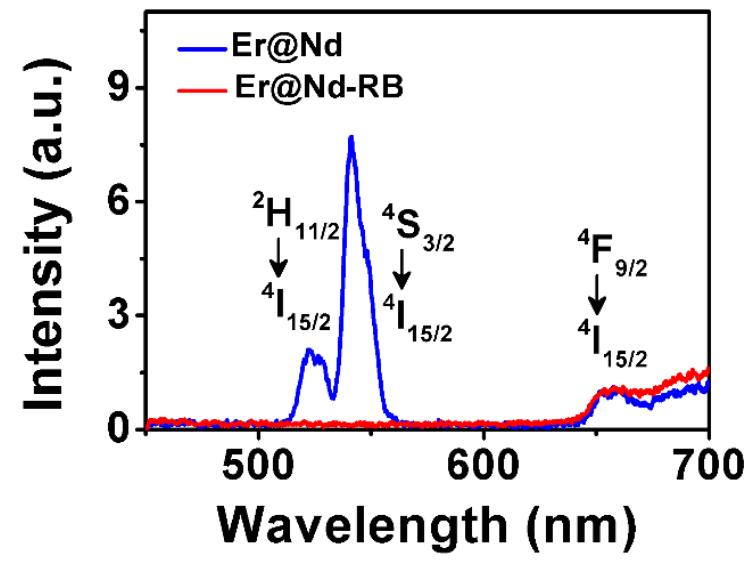

Figure S4. UC emission spectra of the Er@Nd NPs and Er@Nd-RB conjugates normalized at $650 \mathrm{~nm}$. 
Table S1. Fitted lifetime of the Er@Nd NPs and Er@Nd-RB conjugates.

\begin{tabular}{|c|c|c|c|c|c|}
\hline$\lambda_{\mathrm{ex}} / \mathrm{nm}$ & \multicolumn{2}{|c|}{980} & \multicolumn{2}{|c|}{808} & 540 \\
\hline$\lambda_{\mathrm{em}} / \mathrm{nm}$ & 540 & 650 & 540 & 650 & 587 \\
\hline$\tau_{\mathrm{Er} @ \mathrm{Nd}} / \mu \mathrm{s}$ & $121.0 \pm 0.2$ & $200.3 \pm 0.1$ & $128.9 \pm 0.3$ & $217.2 \pm 0.5$ & \\
\hline$\tau_{\mathrm{Er} @ \mathrm{Nd}-\mathrm{RB}} / \mu \mathrm{s}$ & $100.9 \pm 0.1$ & $201.8 \pm 0.2$ & $87.9 \pm 0.5$ & $215.3 \pm 0.3$ & \\
\hline$\tau_{\mathrm{RB}} / \mu \mathrm{s}$ & \multicolumn{2}{|c|}{$74.2 \pm 1.6$} & \multicolumn{2}{|c|}{ - [a] } & $0.8 \pm 0.02(\mathrm{~ns})$ \\
\hline
\end{tabular}

${ }^{\text {[a] }}$ too low to be measured
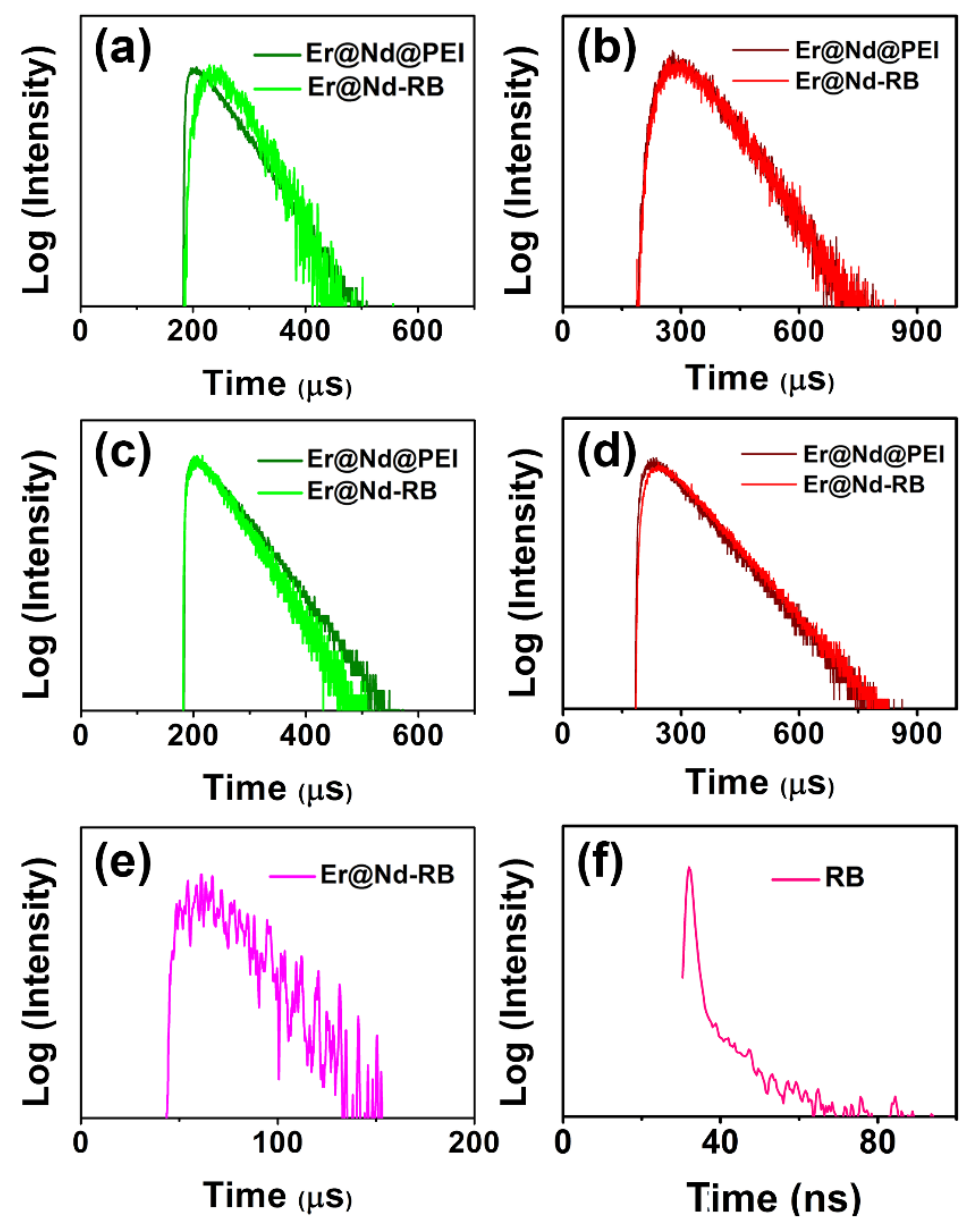

Figure S5. Decay curves of the Er@Nd NPs and Er@Nd-RB conjugates, monitored at $540 \mathrm{~nm}(\mathrm{a}), 650 \mathrm{~nm}$ (b) under $808 \mathrm{~nm}$ excitation. Decay curves of the Er@Nd NPs and Er@Nd-RB conjugates, monitored at $540 \mathrm{~nm}$ (c), $650 \mathrm{~nm}$ (d) under $980 \mathrm{~nm}$ excitation. (e) Decay curve of the Er@Nd-RB conjugates, monitored at $587 \mathrm{~nm}$ under $980 \mathrm{~nm}$ excitation. (f) Decay curve of RB, monitored at $587 \mathrm{~nm}$ under $540 \mathrm{~nm}$ excitation. The excitation source was provided by an OPO pulse laser. 

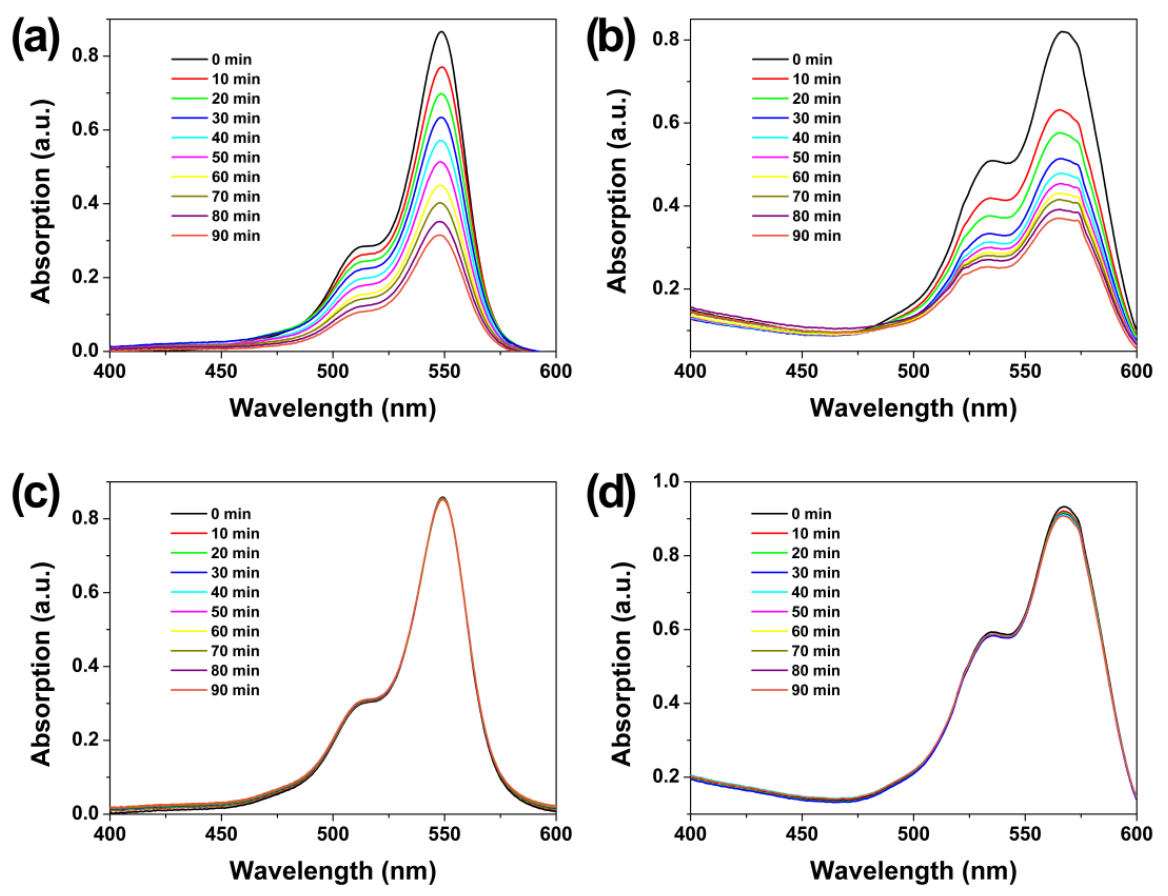

Figure S6. Absorption spectral change of (a) RB and (b) Er@Nd-RB conjugates dispersed in aqueous phase after irradiated by a Xe lamp $\left(45 \mathrm{~mW} / \mathrm{cm}^{2}\right)$, and (c) RB and (d) Er@Nd-RB conjugates dispersed in aqueous phase after irradiated by an $808 \mathrm{~nm}$ laser $\left(1.13 \mathrm{~W} / \mathrm{cm}^{2}\right)$. 

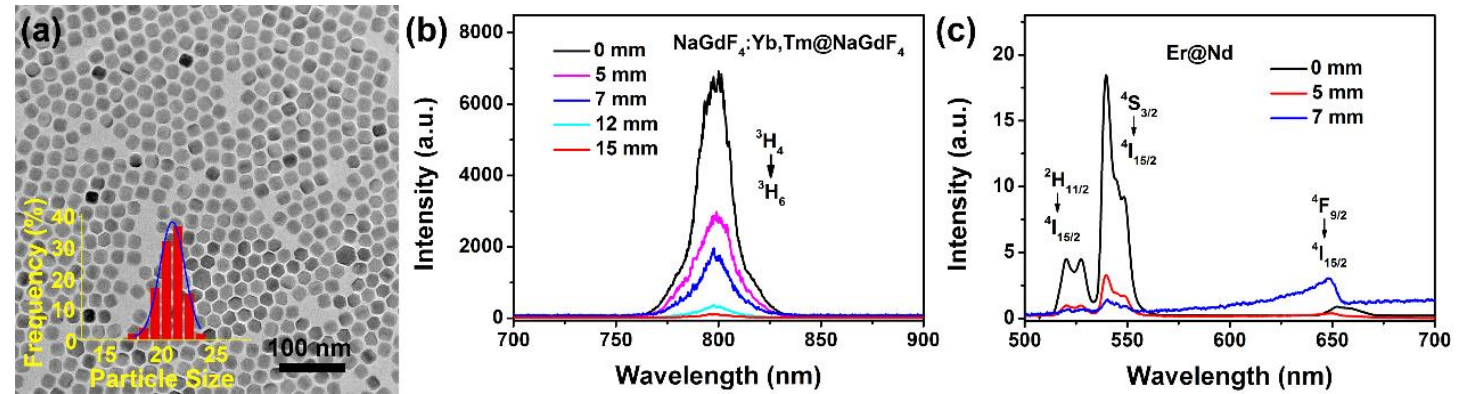

Figure S7. (a) TEM image of $\mathrm{NaGdF}_{4}: \mathrm{Yb}(20 \%), \mathrm{Tm}(0.5 \%) @ \mathrm{NaGdF}_{4} \mathrm{NPs}$. The inset was the particle size statistics of the NPs. (b) UC emission spectra of the

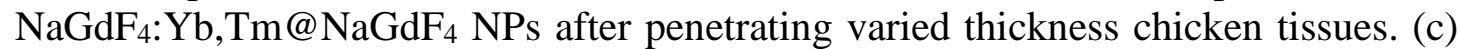
UC emission spectra of the Er@Nd NPs excited at $808 \mathrm{~nm}$. The excitation light penetrated varied thickness chicken tissues to excite the sample. A G2B3 filter was used to block the interference of excitation light.

We further investigated the excitation light on penetration depth which is important for PDT efficiency. In order to mimic the $808 \mathrm{~nm}$ light, $\mathrm{NaGdF}_{4}: \mathrm{Yb}, \mathrm{Tm} @ \mathrm{NaGdF}_{4}(\sim 20 \mathrm{~nm})$ (Figure S11a) with intense $808 \mathrm{~nm}$ emissions were prepared, and the emission signals which we blocked with chicken tissues were collected (Figure S11b). Remarkably, the emission signals could be detected at the depth of $15 \mathrm{~mm}$. This confirmed that the $808 \mathrm{~nm}$ light could penetrate at least $15 \mathrm{~mm}$ for excitation or detection. As a comparison, the influence of the penetration depth of visible light, which comes from the UC emissions of the Er@Nd NPs was studied (Figure S11c). Varied thickness chicken tissues were placed between the $808 \mathrm{~nm}$ excitation source and Er@Nd NPs. At a depth of $5 \mathrm{~mm}$, about $80 \%$ UC green emissions were lost, because of both the excitation light losses and the decreased UC efficiency induced by attenuated excitation power density. 


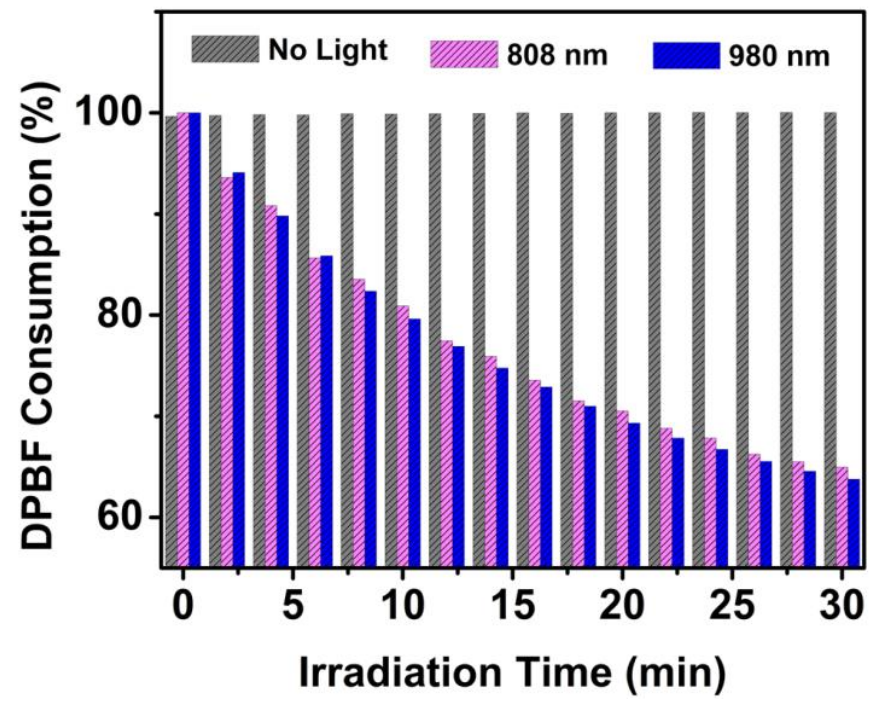

Figure S8. Consumption of DPBF by ${ }^{1} \mathrm{O}_{2} .{ }^{1} \mathrm{O}_{2}$ is generated from the Er@Nd-RB conjugates excited at $808 \mathrm{~nm}$ (violet) and $980 \mathrm{~nm}$ (blue) with the same laser power density of $1.0 \mathrm{~W} / \mathrm{cm}^{2}$. Control experiment was performed without irradiation (dark grey)

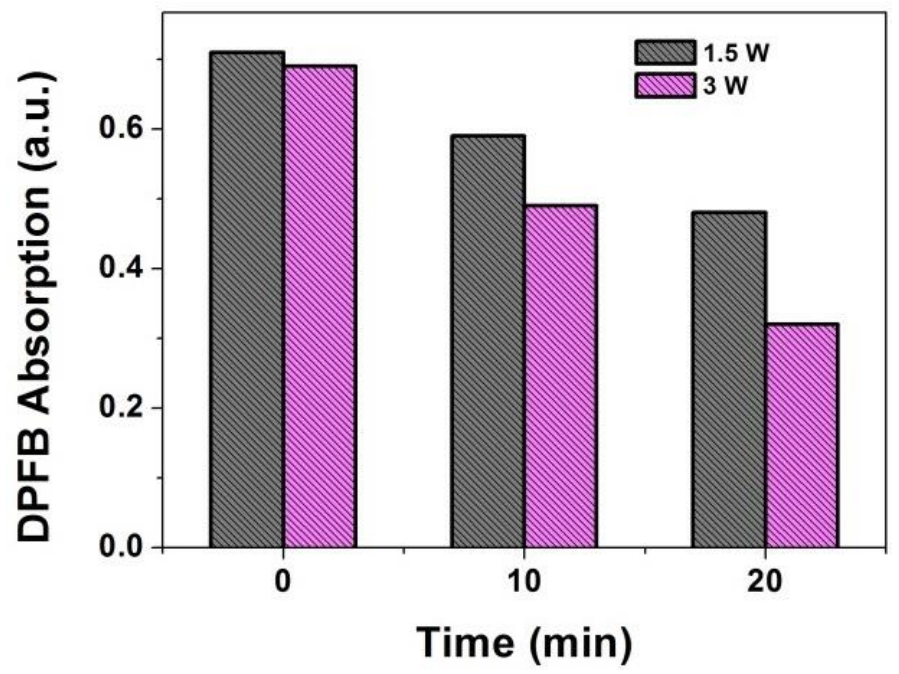

Figure S9. Influence of laser power and irradiation time on the production of ${ }^{1} \mathrm{O}_{2}$. 


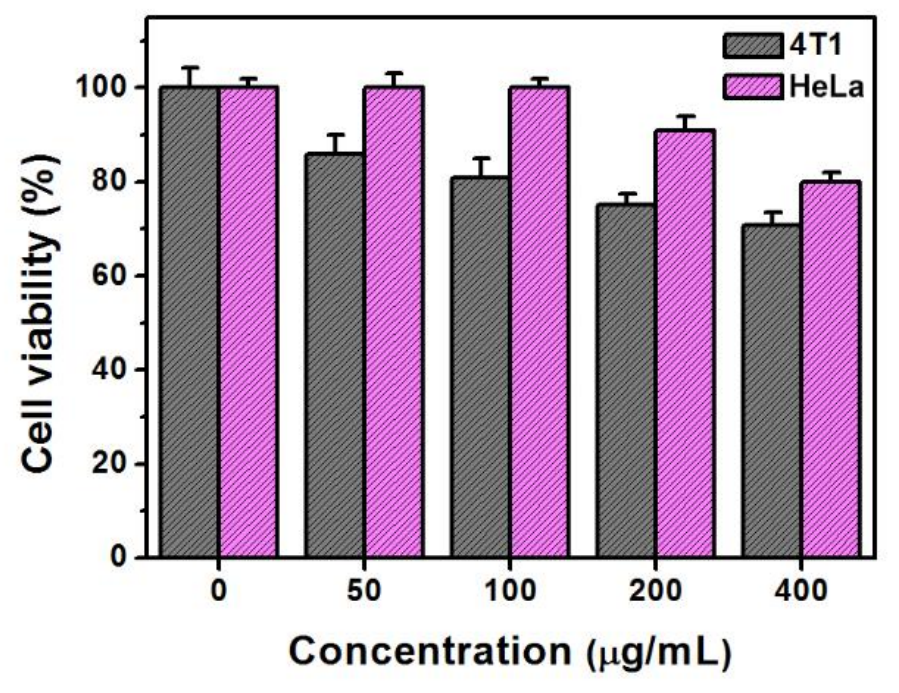

Figure S10. Cell viability of the Er@ Nd-RB conjugates after incubation with 4T1 cells and HeLa cells for $24 \mathrm{~h}$.

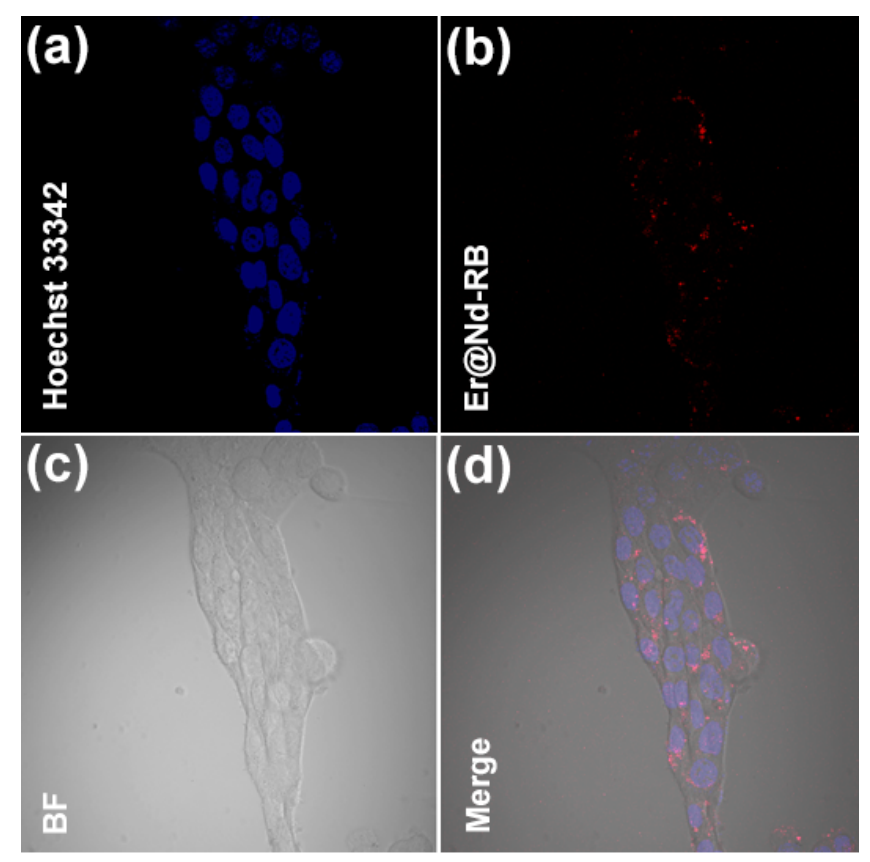

Figure S11. Multiphoton microscopy images of $4 \mathrm{~T} 1$ cells after incubation with the Er@Nd-RB conjugates for 24 h. (a) Image of the Hoechst 33342 stained nuclei (blue channel), excited by a $350 \mathrm{~nm}$ laser. (b) Observed emissions from the Er@Nd-RB conjugates under $980 \mathrm{~nm}$ excitation (red color). (c) Image of the 4T1 cells in bright filed channel. (d) Merged images of the Hoechst 33342, Er@Nd-RB conjugates, and bright field channel together. 

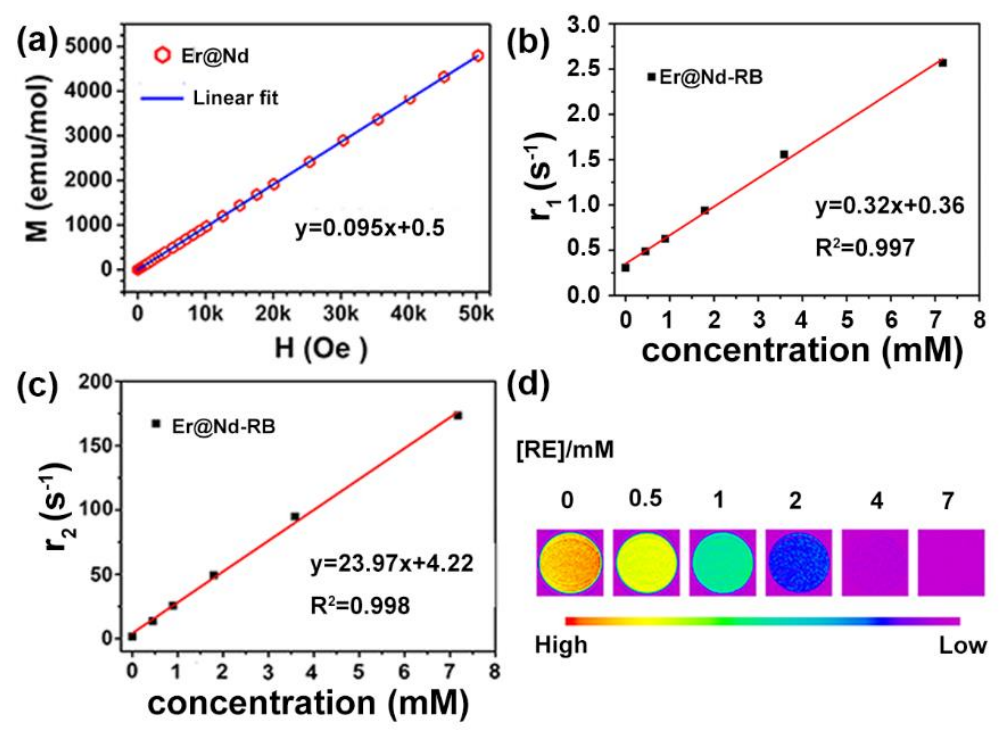

Figure S12. (a) $M-H$ curve of the Er@Nd NPs at $300 \mathrm{~K}$. (b) Relaxation rate $r_{1}\left(1 / T_{1}\right)$ vs. molar concentration of the RE ions in the Er@Nd-RB conjugates at room temperature at $7 \mathrm{~T}$. (c) Relaxation rate $r_{2}\left(1 / T_{2}\right) v s$. molar concentration of the RE ions in the Er@Nd-RB conjugates at room temperature at $7 \mathrm{~T}$. (d) $T_{2}$-weighted color-mapped MRI of various molar concentrations of the RE ions in $\mathrm{Er} @ \mathrm{Nd}-\mathrm{RB}$ conjugates. Deionized water was used as the control.

Besides the unique optical properties, LNPs are also widely applied as $T_{l}$ or $T_{2}$ contrast agents for MRI. ${ }^{\mathrm{S} 1, \mathrm{~S} 2} M-H$ curve revealed the paramagnetism of the Er@Nd NPs (Figure $\mathrm{S} 12 \mathrm{a})$ with the calculated magnetization of $5.24 \mathrm{emu} / \mathrm{g}$. To evaluate the MRI modality of the conjugates, $T_{1}$ - and $T_{2}$-weighted relaxivity parameters $\left(r_{1}\right.$ and $\left.r_{2}\right)$ and images were carried out on a 7 T MRI scanner. The $r_{1}$ and $r_{2}$ for the Er@Nd-RB conjugates were $0.32 \mathrm{mM}^{-1} \cdot \mathrm{s}^{-1}$ (Figure S12b) and $23.97 \mathrm{mM}^{-1} \cdot \mathrm{s}^{-1}$ (Figure S12c), respectively. The large $r_{2} / r_{1}$ of 75 , which was much higher than that of clinically used negative contrast agents such as Combidex, Feridex, and Resovist ( $r_{2} / r_{l}=6,22.6$, and 31, respectively), ${ }^{\mathrm{S} 3, \mathrm{~S} 4}$ suggested these Er@Nd NPs promising high-field $T_{2}$ contrast agents for MRI. The corresponding color-mapped MR images appeared more signal intensity loss with increasing the molar concentration of total RE ions (Figure S12d). 


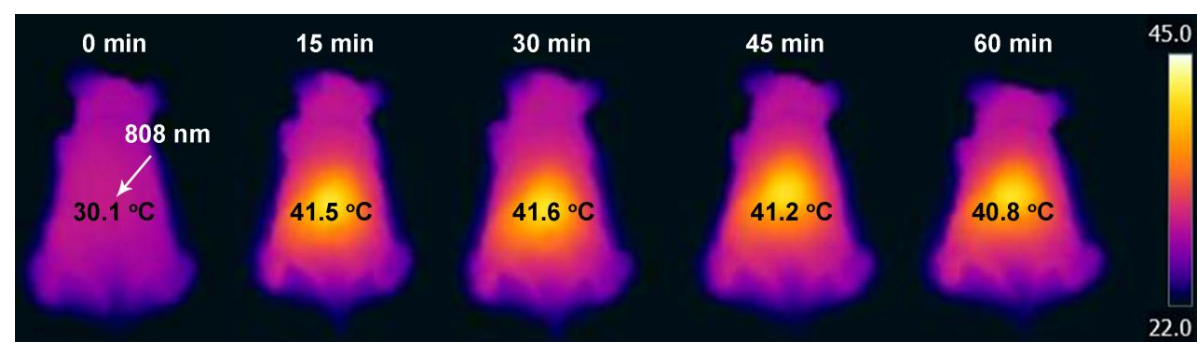

Figure S13. Thermal effect after continuous irradiation on a living nude mouse by an $808 \mathrm{~nm}$ fiber laser $\left(520 \mathrm{~mW} / \mathrm{cm}^{2}\right)$. The array indicates the irradiation site.

Table S2. Blood biochemistry analysis of the mice after treatment. The results show mean and standard deviation of total bilirubin (TBIL), mean and standard deviation of alanine aminotransferase (ALT), aspartate aminotransferase (AST), blood urea nitrogen (BUN), creatinine (CRE), uric acid (UA), total bilirubin (TBIL), creatine kinase (CK).

\begin{tabular}{|c|c|c|c|c|c|c|c|c|}
\hline & $\begin{array}{c}\text { TBIL } \\
(\mu \mathrm{mol} / \mathrm{L})\end{array}$ & $\begin{array}{l}\text { AST } \\
(\mathbf{U} / \mathbf{L})\end{array}$ & $\begin{array}{l}\text { ALT } \\
(\mathbf{U} / \mathbf{L})\end{array}$ & AST/ALT & $\begin{array}{c}\text { BUN } \\
(\mathrm{mmol} / \mathrm{L})\end{array}$ & $\begin{array}{c}\text { Cre } \\
(\mu \mathrm{mol} / \mathrm{L})\end{array}$ & $\begin{array}{c}\text { UA } \\
(\mu \mathrm{mol} / \mathrm{L})\end{array}$ & $\begin{array}{c}\text { CK } \\
(\mathrm{U} / \mathrm{L})\end{array}$ \\
\hline PBS & $6.02 \pm 1.57$ & $90.67 \pm 14.19$ & $8.00 \pm 2.30$ & $12.93 \pm 4.22$ & $5.05 \pm 0.88$ & $27.02 \pm 5.30$ & $151.00 \pm 41.65$ & $290.21 \pm 41.85$ \\
\hline Er@Nd-RB & $6.80 \pm 2.15$ & $88.00 \pm 6.93$ & $8.00 \pm 4.00$ & $14.00 \pm 6.06$ & $4.88 \pm 1.14$ & $37.03 \pm 6.64$ & $144.00 \pm 27.50$ & $239.30 \pm 38.28$ \\
\hline $808 \mathrm{~nm}$ laser & $5.42 \pm 0.78$ & $110.50 \pm 32.80$ & $11.00 \pm 3.60$ & $10.14 \pm 5.44$ & $6.07 \pm 0.71$ & $35.03 \pm 6.22$ & $85.50 \pm 9.98$ & $261.47 \pm 43.55$ \\
\hline PDT & $6.92 \pm 1.55$ & $79.00 \pm 13.90$ & $10.75 \pm 3.40$ & $7.17 \pm 3.94$ & $4.81 \pm 1.02$ & $22.02 \pm 3.46$ & $80.87 \pm 34.02$ & $253.09 \pm 48.50$ \\
\hline Normal & $6.58 \pm 1.40$ & $58.00 \pm 11.89$ & $19.50 \pm 3.41$ & $2.59 \pm 0.71$ & $5.52 \pm 1.04$ & $30.52 \pm 5.26$ & $81.60 \pm 5.90$ & $259.84 \pm 81.47$ \\
\hline
\end{tabular}




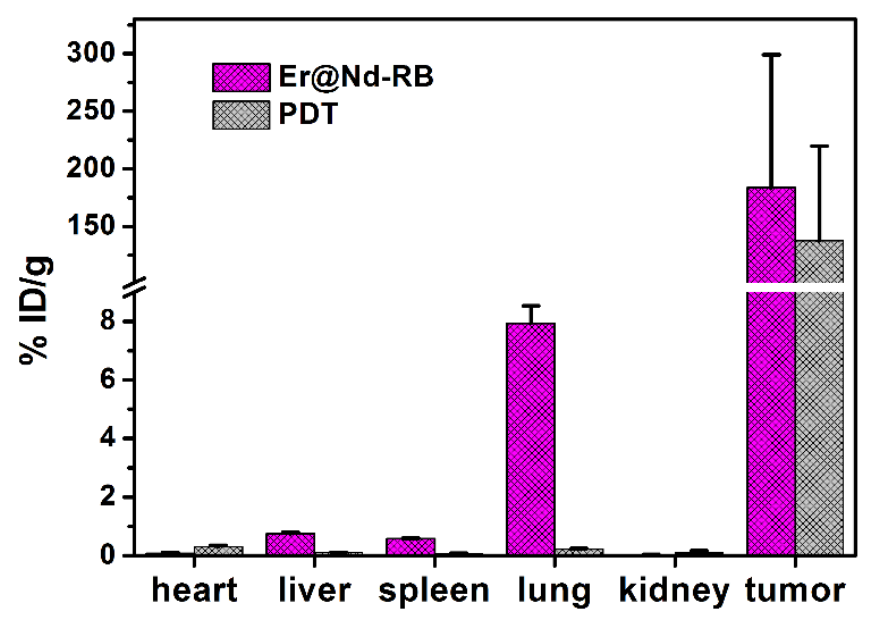

Figure S14. Biodistribution of the Er@Nd-RB conjugates after tumor topical administration. The organ samples were collected at the end of PDT and the concentration of total RE ions was analysis by ICP-AES.

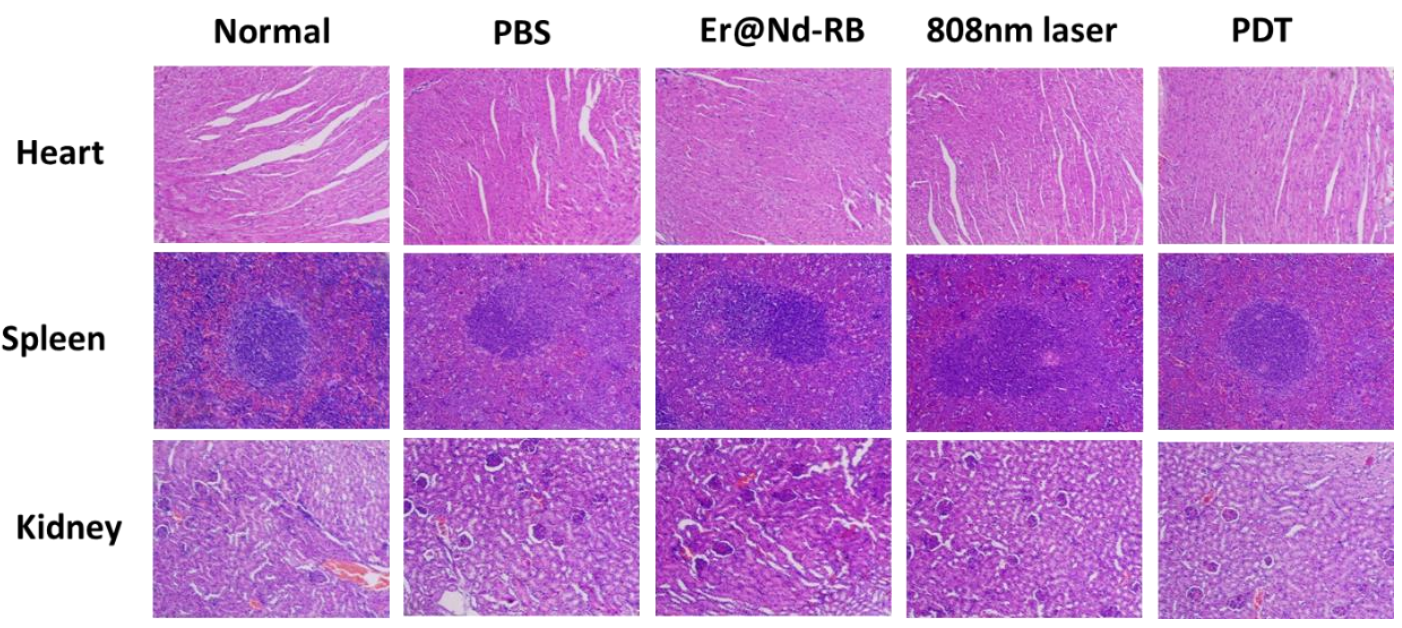

Figure S15. Hematoxylin-eosin $(H \& E)$ staining of tissues after different treatments. Magnification: $\times 200$. 


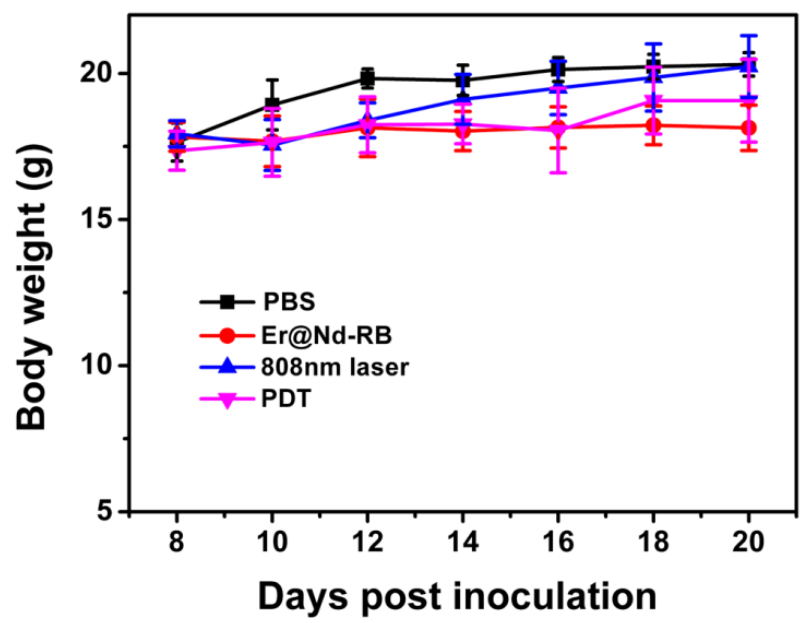

Figure S16. Body weight changes of murine 4T1 tumor-bearing mice after PDT.

\section{Reference}

S1. Dong, H.; Du, S. R.; Zheng, X. Y.; Lyu, G. M.; Sun, L. D. Li, L. D.; Zhang, P. Z.; Zhang, C.; Yan, C. H. Lanthanide Nanoparticles: From Design toward Bioimaging and Therapy. Chem. Rev. 2015, 115, 10725-10815.

S2. Dai, Y.; Xiao, H.; Liu, J.; Yuan, Q.; Ma, P.; Yang, D.; Li, C.; Cheng, Z.; Hou, Z.; Yang, P.; Lin, J. In Vivo Multimodality Imaging and Cancer Therapy by Near-Infrared Light-Triggered trans-Platinum Pro-Drug-Conjugated Upconverison Nanoparticles. $J$. Am. Chem. Soc. 2013, 135, 18920-18929.

S3. Rohrer, M.; Bauer, H.; Mintorovitch, J.; Requardt, M.; Weinman, H. Comparison of Magnetic Properties of MRI Contrast Media Solutions at Different Magnetic Field Strengths. Invest. Radiol. 2005, 40, 715-724.

S4. Wang, Y. Superparamagnetic Iron Oxide Based MRI Contrast Agents: Current Status of Clinical Application. Quant. Imaging Med. Surg. 2011, 1, 35-40. 